${ }^{1}$ Departamento de Enfermedades No Transmisibles. División de Prevención y Control de Enfermedades. Subsecretaria de Salud Pública. Ministerio de Salud. Santiago, Chile. ${ }^{2}$ Facultad de Medicina, Universidad del Desarrollo-Clínica Alemana. Santiago, Chile. ${ }^{a}$ Enfermera. Profesional Asesor.

Recibido el 29 de enero de 2019, aceptado el 8 de mayo de 2019 .

Correspondencia a: Dr. Tomás Labbé Atenas, MD, $\mathrm{PhD}$.

Monjitas 565. Piso 8. Oficina 819. Santiago, Chile. tomas.labbe@minsal.cl

\section{Obesidad: ¿Factor de riesgo o enfermedad?}

\author{
CONSTANZA AGUILERA ${ }^{1}$, TOMÁS LABBÉ ${ }^{1}$, JAVIERA BUSQUETS ${ }^{1}$, \\ PÍA VENEGAS ${ }^{1, a}$, CAROLINA NEIRA $^{1, a}$, ÁLEX VALENZUELA ${ }^{3}$
}

\section{Obesity: risk factor or primary disease?}

Obesity is a global health problem. Its worldwide prevalence has tripled between 1975 and 2016, reaching a prevalence in Chile of 34.4\%, according to the National Health Survey 2016-2017. If this condition corresponds to a risk factor or primary disease is a widely discussed issue. It is recognized as a disease by the American Medical Association and World Health Organization, based on its metabolic and hormonal features, such as dysregulation of appetite, abnormal energy balance and endocrine dysfunction, among others. Its main environmental risk factors are the consumption of ultra-processed foods and sedentariness. Preventive measures at the population level are fundamental, emphasizing promotion and prevention using a transdisciplinary approach. The individual approach in the management of obesity should improve the quality of life, avoid early mortality, reduce cardiovascular risk, and reduce the progression to type 2 diabetes and incidence of cancer. Thus, an adequate management and control of obesity would have a great impact in our society.

(Rev Med Chile 2019; 147: 470-474)

Key words: Metabolic Syndrome; Obesity; Preventive Medicine; Public Health.

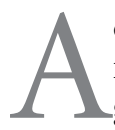
ctualmente, de acuerdo a la clasificación internacional de enfermedades de la Organización Mundial de la Salud (OMS), la obesidad se define como el anormal o excesivo almacenamiento de grasa, secundario a diferentes causas, incluyendo desbalance energético, fármacos y patología genética ${ }^{1}$. La Federación Mundial de Obesidad (World Obesity Federation [WOF]) la ha definido como una enfermedad crónica, recurrente y progresiva, enfatizando en la necesidad de acción inmediata para la prevención y control de esta ${ }^{2}$.

Este problema sanitario constituye una epidemia global. De acuerdo a la OMS, la prevalencia a nivel mundial se ha triplicado entre 1975 y 2016, alcanzando cifras de más de 1,9 billones de adultos de 18 años o más con sobrepeso, de los cuales, 650 millones eran obesos, representando $13 \%$ de la población adulta a nivel mundial ${ }^{3,4}$. Así, la población ha incrementado su índice de masa corporal
(IMC) en $1 \mathrm{~kg} / \mathrm{m}^{2}$ por década. La obesidad infantil también es una realidad. A nivel mundial, la prevalencia aumentó de $0,7 \%$ a $5,6 \%$ en niñas, y de $0,9 \%$ a $7,8 \%$ en niños, alcanzando 50 millones y 74 millones, respectivamente, para el año $2016^{5}$. En Chile, la Encuesta Nacional de Salud (ENS) de 2016-2017, revela que $31,2 \%$ de las personas adultas mayores de 15 años son obesas y 3,2\% obesas mórbidas, mientras que $39,8 \%$ son clasificadas con sobrepeso, generando una realidad sanitaria alarmante 6 . Mientras, la población menor de 6 años también se ha visto afectada, aumentando la prevalencia de malnutrición por exceso de $7,1 \%$ a $10,3 \%$ entre los años 2005 y $2013^{7}$.

\section{Obesidad ¿factor de riesgo o enfermedad primaria?}

Si esta condición corresponde a un factor de riesgo o a una enfermedad es un tema que genera 
discusión. Similares debates se han dado en otros casos, como la osteoporosis, que pasó de ser considerada un componente normal de envejecimiento a ser reconocida como enfermedad por la OMS en $1994^{8}$

Respecto a obesidad, para enfrentar esta pregunta es esencial definir qué entendemos como factor de riesgo y enfermedad. Según la OMS, enfermedad corresponde a "alteración o desviación del estado fisiológico en una o varias partes del cuerpo, por causas, en general, conocidas, manifestada por síntomas y signos característicos, y cuya evolución es más o menos previsible" ${ }^{\text {. La }}$ Asociación Médica Americana (American Medical Association [AMA]) adoptó los siguientes criterios comunes para definir enfermedad: falla del funcionamiento normal de algún sistema corporal, signos y síntomas característicos y daño o morbilidad ${ }^{10}$.

Por otro lado, factor de riesgo se comprende como cualquier atributo, característica y exposición de un individuo que aumente la probabilidad de desarrollar una enfermedad o lesión, como son los casos del tabaco o promiscuidad sexual ${ }^{11}$.

Desde el punto de vista epidemiológico, la enfermedad resulta de la interacción entre un agente ambiental y un huésped. El resultado será producto de la virulencia del agente y la susceptibilidad del huésped. Los alimentos ultraprocesados, principalmente aquellos ricos en grasas, azúcares y sal, altamente palatables e incluso para algunos adictivos, en conjunto con la inactividad física, favorecida por la tecnificación, son los dos principales agentes ambientales responsables de la alta prevalencia de obesidad a nivel mundial ${ }^{2}$. Otros agentes ambientales incluyen fármacos obesogénicos, toxinas, estrés, infecciones y privación de sueño, entre muchos otros, produciendo un desbalance energético crónico a favor de los ingresos calóricos con respecto al gasto, expresándose por una acumulación de grasa anormal e incremento progresivo del peso corporal ${ }^{2,12}$. En 1997, Maes et al. sugirieron factores genéticos que explicaban, en parte, la variación del $\mathrm{IMC}^{13}$. Posteriormente, el estudio para la secuenciación completa del genoma humano (GWAS) ha identificado al menos 52 loci relacionados con obesidad y la susceptibilidad a esta ${ }^{14}$. Agregado a lo anterior, factores epigenéticos, tales como diabetes gestacional, se relacionan al sobrepeso en la adolescencia ${ }^{15}$. De esta forma, el modelo complejo considera factores modificables, tales como ingesta alimentaria y actividad física, y otros no modificables, como la herencia ${ }^{16}$. Así, comprendemos el modelo epidemiológico para la obesidad como un proceso crónico progresivo en que un agente ambiental actúa sobre un huésped para producir enfermedad ${ }^{2}$.

Así, el concepto de obesidad ha pasado de ser considerada, más que un factor de riesgo, a una enfermedad primaria, proceso que no ha estado exento de discusiones. Han sido numerosas las publicaciones en que sociedades internacionales invitan a identificarla como enfermedad. En 2008, The Obesity Society (TOS) publicó un documento de posición que define la obesidad como una enfermedad ${ }^{17}$. Posteriormente, en 2013, la AMA reconoció la obesidad como una enfermedad ${ }^{10}$, seguido en breve por otras organizaciones y sociedades, como la OMS, Food and Drug Administration (FDA) de los Estados Unidos de Norteamérica, National Institutes of Health (NIH), American Association of Clinical Endocrinologists (AACE), Internal Revenue Service (IRS) y recientemente la WOF, todas las cuales también posicionan a la obesidad como una enfermedad ${ }^{2,18}$. Algunas de las justificaciones empleadas por la AMA son el carácter multimetabólico y hormonal, incluyendo disregulación del apetito, balance energético anormal, disfunción endocrina (elevados niveles de leptina e insulino resistencia), infertilidad y función endotelial anormal, entre otras ${ }^{10}$. Además, síntomas y signos como dolor articular, inmovilidad y apnea del sueño, que proveen un cuadro clínico que permite la caracterización semiológica. Adicionalmente, la AACE ha enfatizado la necesidad de verla como un desorden crónico, que requiere cuidado permanente, apoyo y seguimiento ${ }^{18}$. La Figura 1 muestra diferentes mecanismos relacionados con la obesidad como una enfermedad.

No obstante lo anterior, se ha sugerido que la catalogación de la obesidad como una enfermedad desplazaría la responsabilidad de la baja de peso desde el individuo a la sociedad, aumentando el gasto médico y sus costos de prevención y tratamiento ${ }^{19}$. Por otro lado, se ha cuestionado la definición de obesidad como enfermedad basada tan solo por un punto de corte en el valor de IMC, comprendiendo que el proceso de estudio médico debe ser más amplio y riguroso, incluyendo examen físico, historia clínica detallada y parámetros de laboratorio, tales como: glicemia, lipidemia, 


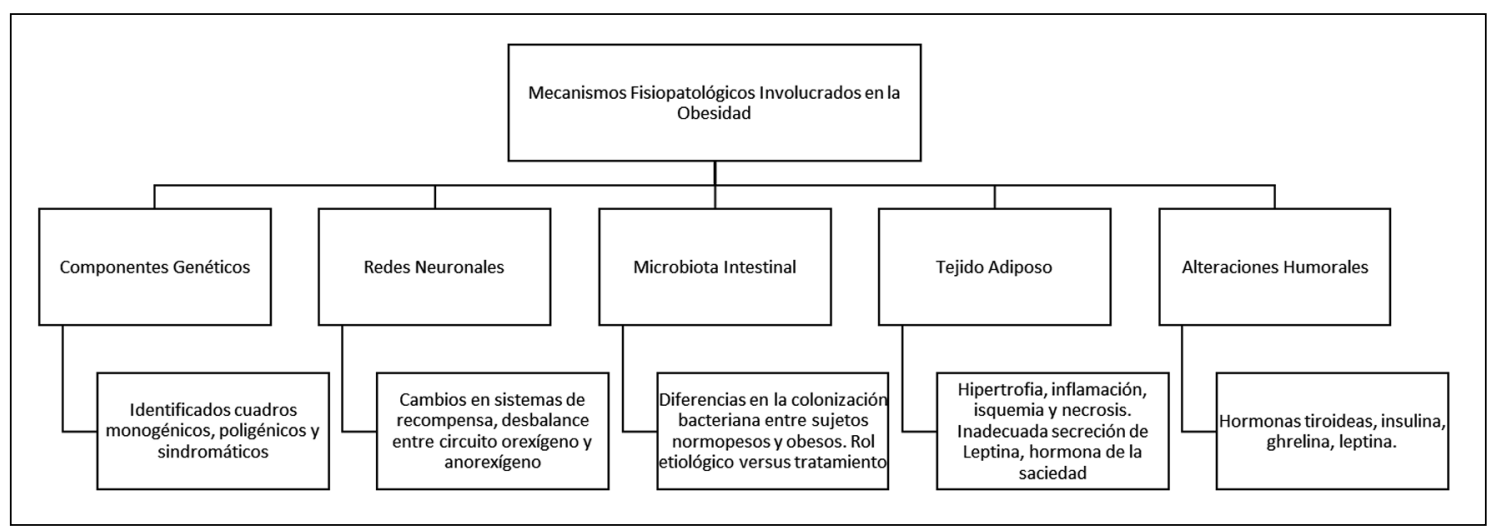

Figura 1. Mecanismos fisiopatológicos involucrados en la obesidad. Entre otros factores, los aquí mencionados refuerzan el enfoque de la obesidad como una enfermedad desde la perspectiva de sus diversos componentes objetivables desde la aproximación biomédica.

mediciones hormonales y electrocardiograma. Además, debe tenerse presente que existe un subgrupo de pacientes obesos (IMC $\geq 30 \mathrm{~kg} / \mathrm{m}^{2}$ ) que al momento de su diagnóstico no presentan patologías asociadas a la obesidad, definiéndose como obesos metabólicamente saludables. Por tanto, se ha enfatizado que la decisión debe ser basada en un perfil completo del paciente y no tan solo en su IMC ${ }^{20}$.

Resulta interesante el concepto de problema que envuelve la interacción individual con una "sociedad obesogénica" ${ }^{20}$, concepto que nos recuerda que se requieren medidas poblacionales, tales como promoción y prevención. Al respecto, importantes han sido las medidas llevadas a cabo por el Ministerio de Salud de Chile, enfocadas a regular entornos alimentarios, tales como Ley de Alimentos, Ley de Publicidad de los Alimentos, impuesto al azúcar en la reforma tributaria, impuesto a las bebidas azucaradas, estrategias de promoción de la salud en comunas e implementación de mecanismos de participación ciudadana en materia de alimentación y nutrición ${ }^{21}$.

En Chile, se calcula que los costos derivados de la obesidad alcanzaron $0,54 \%$ del producto interno bruto (PIB) en el año 2016, y se estima que un trabajador obeso resulta seis veces más caro que uno normopeso, debido a factores como ausentismo laboral y pérdida de productividad ${ }^{22,23}$. Lo anterior evidencia el impacto de la condición no tan solo en salud, sino también en sectores distintos a esta. Por tanto, es imperativo que estas medidas deben ser estructurales, poblacionales y comprender trabajo intersectorial ${ }^{24,25}$, comprometiendo entidades del ámbito laboral, social, educacional e industrial, entre otros.

Además del enfoque poblacional, la centralización individual sobre el paciente que padece obesidad requiere acciones aplicadas, destinadas a la reducción de peso corporal, que puedan mejorar su calidad de vida y disminuir radicalmente la mortalidad temprana, progresión a la diabetes mellitus tipo 2, riesgo cardiovascular, mayor incidencia de algunas neoplasias, e incluso, llegar a constituir por su intermedio un tratamiento efectivo para patologías asociadas presentes, como diabetes mellitus tipo 2 o hipertensión arterial ${ }^{10}$.

Probablemente, la concepción de obesidad como enfermedad favorecerá la gestión de recursos para prevención, tratamiento e investigación, contribuyendo a reducir el estigma y discriminación de muchas personas con obesidad ${ }^{26,27}$. Hasta que sea reconocida universalmente como una enfermedad crónica y no una elección de estilo de vida, será poco probable la reducción de su prevalencia ${ }^{28}$.

\section{Conclusión}

En la obesidad coexisten los componentes de una enfermedad primaria y un factor de riesgo, de acuerdo a su relación con otras patologías. La propuesta de un modelo epidemiológico de enfermedad que la explica no ha estado exenta de discusiones, pero ha sido cada vez más reconocida 
como tal por diversas entidades académicas, médicas, científicas e instituciones de salud, logrando un consenso mayoritario a nivel mundial.

En Chile, es imprescindible alinearse con las entidades internacionales e identificarla como enfermedad primaria, de carácter crónico. Esto permitiría enfocarnos en su diagnóstico y tratamiento, sin olvidar las medidas más costo-efectivas: promoción y prevención a lo largo del ciclo vital. Así, nos encaminaremos a la reducción de su prevalencia, la cual requiere de trabajo intersectorial de distintas entidades relacionadas al ámbito educacional, laboral, social, económico, deportivo y de vivienda, entre otros. Grandes tareas para poder alcanzar la meta de control de impacto poblacional e individual.

\section{Referencias}

1. Organización Mundial de la Salud. Clasificación internacional de enfermedades para estadísticas de mortalidad y morbilidad. Décimo primera revisión (ICD-11). Capítulo 5: Enfermedades endocrinas, nutricionales o metabólicas. Disponible en: https://icd.who.int/ browse11/l-m/en\#/http\%3a\%2f\%2fid.who.int\%2ficd\%2fentity\%2f14940304. [Consultado el 23 de enero de 2019].

2. Bray GA, Kim KK, Wilding JPH, World Obesity Federation. Obesity: a chronic relapsing progressive disease process. A position statement of the World Obesity Federation. Obes Rev 2017; 18 (7): 715-23.

3. Organización Mundial de la Salud. "Noncommunicable Diseases Country Profiles 2018”. Disponible en: https:// www.who.int/nmh/publications/ncd-profiles-2018/en/. [Consultado el 23 de enero de 2019].

4. Organización Mundial de la Salud. Obesity and overweight. Disponible en: https:/www.who.int/news-room/ fact-sheets/detail/obesity-and-overweight [Consultado 22 de enero de 2019]. Disponible en: https://www.who. int/news-room/fact-sheets/detail/obesity-and-overweight

5. Abarca-Gómez L, Abdeen ZA, Hamid ZA, Abu-Rmeileh $\mathrm{NM}$, Acosta-Cazares B, et al. Worldwide trends in bodymass index, underweight, overweight, and obesity from 1975 to 2016: a pooled analysis of 2416 population-based measurement studies in 128.9 million children, adolescents, and adults. Lancet 2017; 390 (10113): 2627-42.

6. Ministerio de Salud. Encuesta Nacional de Salud 20162017: primeros resultados. Disponible en: https://www. minsal.cl/wp-content/uploads/2017/11/ENS-2016-
17_PRIMEROS-RESULTADOS.pdf [Consultado 23 de Enero de 2019].

7. Ministerio de Salud. Diagnóstico del estado nutricional de menores de 6 años, gestantes, nodrizas y adultos mayores, bajo control en el sistema público de salud. Diciembre 2013. Disponible en: https://www.minsal.cl/ sites/default/files/DIAGNOSTICO_ESTADO_NUTRICIONAL_DICIEMBRE_2013.pdf. [Consultado 23 de enero de 2019].

8. Kanis JA, Melton LJ, Christiansen C, Johnston CC, Khaltaev N. The diagnosis of osteoporosis. J Bone Miner Res 1994; 9 (8): 1137-41.

9. Universidad de Córdoba. Enfermedad: información del término. Disponible en http://www.uco.es/servicios/ dgppa/images/prevencion/glosarioprl/fichas/e/Enfermedad.html [Consultado el 17 de Enero de 2019].

10. American Medical Association House Of Delegates. Recognition of Obesity as a Disease. Resolution: 420 (A-13). Disponible en: https://www.npr.org/documents/2013/jun/ama-resolution-obesity.pdf [Consultado el 23 de enero de 2019].

11. Organización Mundial de la Salud. Health topics: Risk Factor. Disponible en: https://www.who.int/topics/ risk_factors/en/ [Consultado el 23 de enero de 2019].

12. Bray GA. Obesity: The Disease. J Med Chem 2006; 49 (14): 4001-7.

13. Maes HH, Neale MC, Eaves LJ. Genetic and environmental factors in relative body weight and human adiposity. Behav Genet 1997; 27 (4): 325-51.

14. Loos RJF. Genetic determinants of common obesity and their value in prediction. Best Pract Res Clin Endocrinol Metab 2012; 26 (2): 211-26.

15. Gillman MW, Rifas-Shiman S, Berkey CS, Field AE, Colditz GA. Maternal gestational diabetes, birth weight, and adolescent obesity. Pediatrics 2003; 111 (3): e221-6.

16. Bray GA. Prevention of Obesity. De Groot LJ, Chrousos G, Dungan K, Feingold KR, Grossman A, Hershman JM, et al., editores. Endotext (Internet). South Dartmouth (MA): MDText.com, Inc.; 2000. Disponible en: http:// www.ncbi.nlm.nih.gov/books/NBK279120/ [Consultado el 11 de enero de 2019].

17. Council of the Obesity Society. Obesity as a disease: the Obesity Society Council resolution. Obesity (Silver Spring) 2008; 16 (6): 1151.

18. Garvey WT, Garber AJ, Mechanick JI, Bray GA, Dagogo-Jack S, et al, for the AACE Obesity Scientific Committee. American Association of Clinical Endocrinologists and American College of Endocrinology consensus conference on obesity: building an evidence base for comprehensive action. Endocr Pract 2014; 20: 956-76.

19. Tanner M. Obesity is not a disease. National Review On- 
line. 2013. Disponible en: http://www.nationalreview. com/article/352626/obesity-not-disease-michael-tanner. [Consultado el 8 de enero de 2019].

20. Kabat G. Why labeling obesity as a disease is a big mistake (Internet). Forbes. Disponible en: https://www. forbes.com/sites/geoffreykabat/2013/07/09/why-labeling-obesity-as-a-disease-is-a-big-mistake/ [Consultado el 9 de enero de 2019].

21. Ministerio de Salud. Balance de gestión: División de Políticas Públicas Saludables y Promoción 2014-2018. Página 31. Diciembre 2017. Disponible en: https:// www.minsal.cl/wp-content/uploads/2018/03/Adicional-SSP-DIPOL.-Balance-de-Gesti\%C3\%B3n.pdf. [Consultado el 23 de enero de 2019].

22. Yáñez C. Estudio calcula que este año Chile gastará $0,5 \%$ del PIB por causa de la obesidad. La Tercera Internet. Disponible en: https://www.latercera.com/noticia/ estudio-calcula-este-ano-chile-gastara-05-del-pib-causa-la-obesidad/ [Consultado el 22 de enero de 2019].

23. A 2030 se estiman 14.780 muertes a causa de sobrepeso y obesidad - Escuela de Salud Pública - Universidad de Chile (Internet) (citado el 22 de enero de 2019). Disponible en: http://www.saludpublica.uchile.cl/ noticias/128966/a-2030-se-estiman-14780-muertes-a- causa-de-sobrepeso-y-obesidad.

24. Consulta mixta OMS/FAO de expertos en régimen alimentario, nutrición y prevención de enfermedades crónicas: Dieta, nutrición y prevención de enfermedades crónicas: informe de una Consulta Mixta de Expertos OMS/FAO. OMS, Serie de informes técnicos; 916. 2003.

25. Cruz Sánchez M, Tuñón Pablos E, Villaseñor Farías M, Álvarez Gordillo G, Byronet R, et al. Sobrepeso y obesidad: una propuesta de abordaje desde la sociología. Región y sociedad 2013; 25 (57): 165-202. Disponible en: http://www.scielo.org.mx/scielo.php?script=sci_arttext\&pid=S1870-39252013000200006\&lng=es\&tlng=es. [Consultado el 21 de enero de 2019].

26. Allison DB, Downey M, Atkinson RL, Billington CJ, Bray GA, et al. Obesity as a Disease: A White Paper on Evidence and Arguments Commissioned by the Council of The Obesity Society. Obesity 2008; 16 (6): 1161-77.

27. Kyle TK, Dhurandhar EJ, Allison DB. Regarding Obesity as a disease: Evolving policies and their implications. Endocrinol Metab Clin North Am 2016; 45 (3): 511-20.

28. Editorial The Lancet Diabetes \& Endocrinology. Should we officially recognise obesity as a disease? Lancet Diabetes Endocrinol 2017; 5 (7): 483. 